\title{
Developing updated physical optics curriculum: incorporating the neglected reality of non-interaction of waves (NIW)
}

\section{ChandraSekhar Roychoudhuri}

ChandraSekhar Roychoudhuri, "Developing updated physical optics curriculum: incorporating the neglected reality of non-interaction of waves (NIW)," Proc. SPIE 11143, Fifteenth Conference on Education and Training in Optics and Photonics: ETOP 2019, 1114300 (2 July 2019); doi: 10.1117/12.2520174 


\title{
Developing updated physical optics curriculum: Incorporating the neglected reality of Non-Interaction of Waves (NIW)
} ChandraSekhar Roychoudhuri

Physics Department, University of Connecticut, Storrs, CT, USA 06269

\begin{abstract}
Huygens-Fresnel diffraction integral is the foundation for optical science and engineering since 1817, including the mathematical improvements. This integral propagates Huygens' individual secondary wavelets unperturbed through each other. Huygens postulated this Non-Interaction of Waves (NIW) in his 1690 book, Treatise on Light. Quantum Electrodynamics also indicates NIW since the computed photon-photon interaction cross-section is immeasurably small. The linear sum of complex amplitudes does not represent an observable phenomenon. Fringes represent the energy absorbed by a detector as the square modulus of the sum of amplitude stimulation experienced by it. The detectors' intrinsic properties determine the observable fringes. Due to our neglect of Huygens' NIW, we are carrying on erroneous interpretations for several major optical phenomena. Our proposed experiment-guided curriculum will rectify the confusions. The new book will be developed using the currently published reference book, "Causal Physics; Photon by Non-Interaction of Waves”, Taylor \& Francis, 2014 [1].

Keywords: Non-Interaction of Waves, NIW, Superposition Principle, Superposition Effect, Causal Physics, Hybrid Photon, Semi-classical model, Locality of superposition effect.

\section{INTRODUCTION}

We need an optics textbook for the senior level students, which corrects the multi-century old neglect of the fundamental property of all propagating waves. Propagating waves, by themselves, do not interfere to reorganize their energy distribution to generate the "interference fringes". It is the response characteristics of quadratic detectors that generate the observable fringes. This quadratic response is the observable Superposition Effect. Superposition Principle, the mathematical linear summation of multiple complex-amplitudes, is not an observable phenomenon. It is only a mathematical statement of their co-propagations. Recognition of Non-Interaction of waves (NIW) will open up many innovations and invention potential in the field of classical and quantum optics.
\end{abstract}

\subsection{Introducing Non-Interaction of Waves (NIW)}

Propagating waves have characteristic Poynting vectors that are orthogonal to every point on the diffractively spreading wavefront. The stars being very very far from the earth, our telescopes can capture only a tiny plane-wave section of the spatial wavefront out of any star. These plane wave segments from different stars, co-propagating through each other for multiple light-years, hit our telescope with their Poynting vectors that are at different angles with each other. When we align the telescope with one of the specific Poynting vector, we can image that particular star on axis, along with surrounding ones that are within the broad view of the telescope. Astrophysicists carry out spectroscopic and other detailed studies about the nature of each star independent of others, even though their wave fronts have been propagating through each other over a very long distance. EM waves do not influence any of each other's individual wavecharacteristics (properties). We do not explicitly recognize this Non-Interaction of Wave (NIW) in standard textbooks.

Consider again the principle behind the technology of wavelength domain multiplexing (WDM). A good number of optical beams of multiple different wavelengths, each modulated with its own unique data, can be "spectrometrically" multiplexed into a single collinear beam and transported through one single mode fiber to a distant geographical location. At the output end, one de-multiplexes the output beam into independent channel. Data in individual demultiplexed channel remain un-perturbed in spite of long co-propagation. This another example of NIW that empowers our global internet technology.

An Arab physicist, Alhazen, experimentally demonstrated the NIW-property of light about one thousand years ago. The concept was formally re-introduced by Huygens in his 1690 book, Treatise on light, where he proposed his nowfamous diffractive propagation of light through secondary wavelets emanating out of every point of an optical 
wavefront. Modern physics also repeatedly re-discovered NIW, but ignored it in favor of the persistent cultural belief in "Interference of light", even though light does not interfere with light. They pass through each other unperturbed in the absence of interacting material. For a brief history of the recognition of NIW, repeatedly stumbling upon NIW, and their benign modern neglect, see ref.2 [2].

\subsection{Wide implications of NIW in all physics}

Regarding propagation of light waves, Huygens [3] philosophical approach was distinctly different from that of Newton. Huygens thought through the possible physical processes behind the perpetual velocity of light. Recognized the need for all permeating electromagnetic tension field to allow the perpetual velocity of light through the entire cosmic medium. This process-driven thinking, or the Interaction Process Mapping Epistemology (IPM-E), allowed him to visualize the non-interacting secondary wavelet model that emanate through every point on a wavefront and expand out as spherical wavefronts [see Chart 1 below].

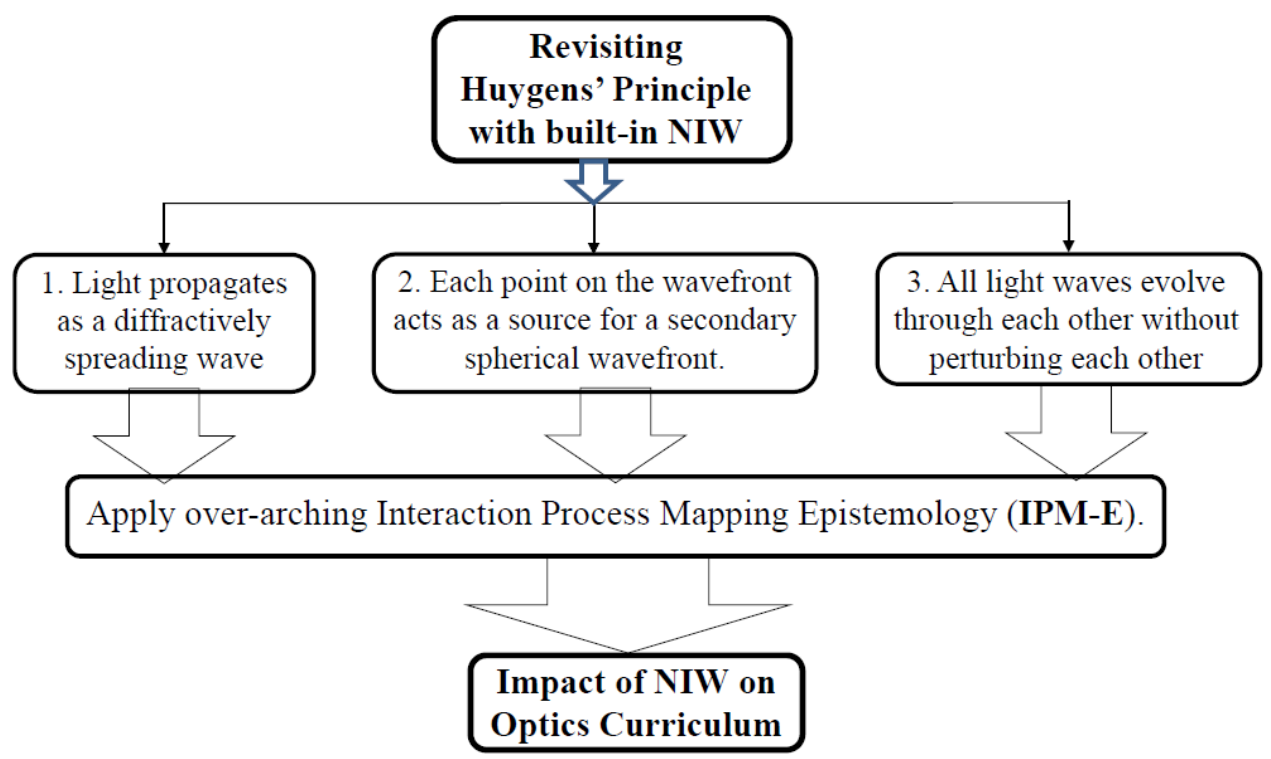

Chart 1. Huygens elaborated wave propagation by visualizing the physical processes behind light propagation. (1) Light propagates as a diffractively spreading wave. (2) Each point on the wavefront acts as a source for a secondary spherical wavelet. And (3) all light waves evolve through each other without interacting, or perturbing each other's fundamental characteristics, which we call, Non-Interaction of Waves or NIW. [See next chart for continuation of the presentation.].

During 1817, Fresnel gave a mathematical structure to Huygens Principle of light propagation, now known as Huygens-Fresnel diffraction integral (HF-DI). The integral literally sums infinite number of spherical wavelets, expanding through each other, independent of each other and without interacting with each other. In 1864, Maxwell formulated his wave equation. It was clear that this second-order differential equation accepts any linear combination of all possible solutions of the equation. Thus, the HF-DI, the non-interaction sum of individual spherical waves, is also a solution of Helmholtz homogeneous wave equation. Non-interaction of waves, or NIW, is an inherent property of all propagating waves.

Unfortunately, Young's double-slit experiment presented in 1802, as interference of light, rather than Superposition Effect (SE) as perceived by our stimulated retinal molecules, and absorbing energy out of both the superposed beams while executing the quantum mechanical square modulus operation during the electronic energy level transition. Of course, such processes behind our "seeing light" were not known. Therefore, the phrase, Interference of Light, and the concomitant belief that light waves interact with light 
waves attained the status of hard science. This is in spite of the facts that the structure of our mathematics were telling us otherwise.

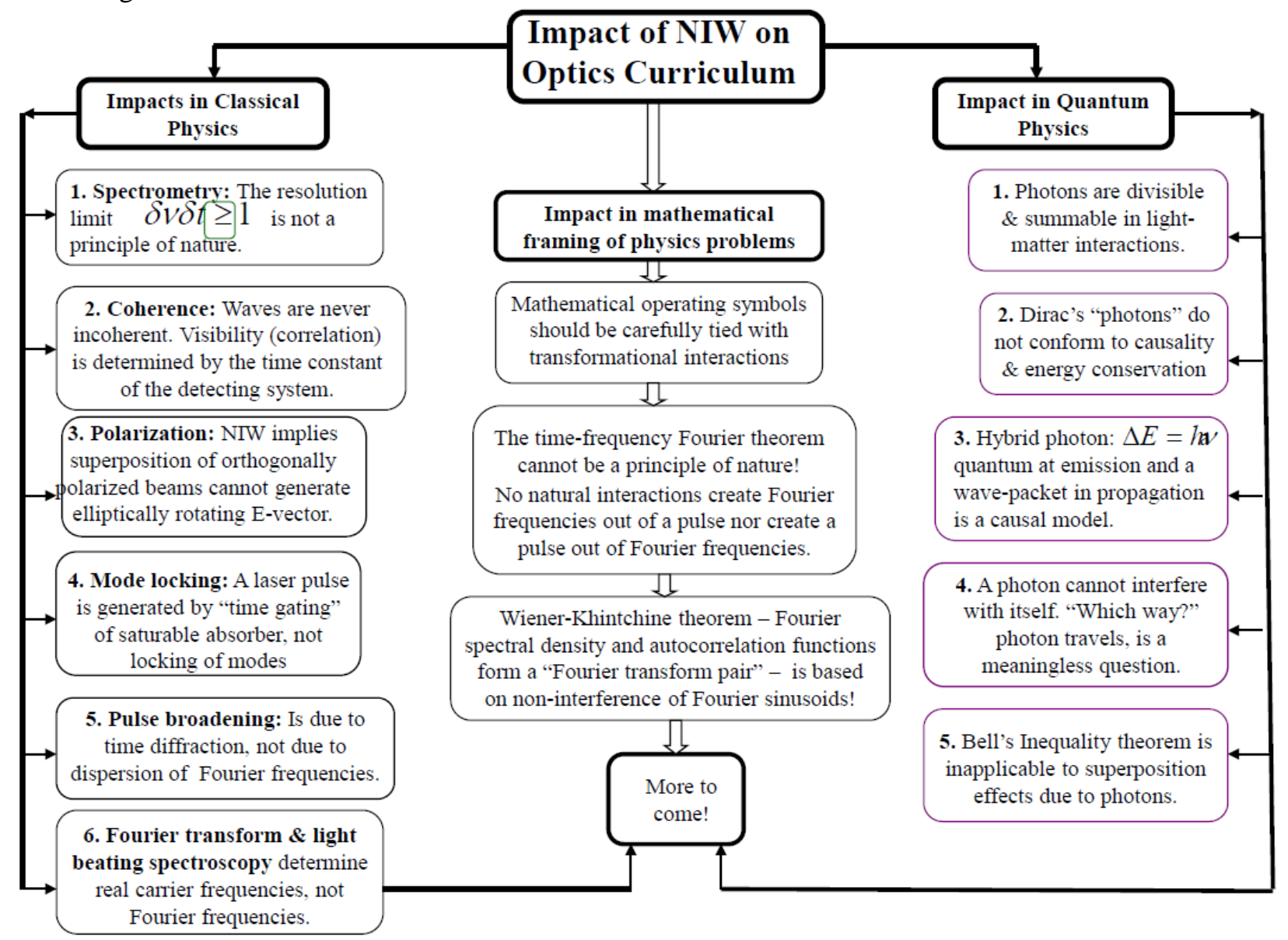

\section{Chart 2. Impact of NIW: This flow diagram briefly summarizes some of the confusing and/or inaccurate interpretation of a good number of optical phenomena in classical, mathematical and quantum optics.}

The Chart 2 gives a brief summary of the necessary conceptual changes, along with interpretational and re-formulation needs in the broad field of optical science and engineering. For deeper implications in all branches physics, consult the 2019 ETOP conference paper Proc. SPIE. Vol.11143-65 (2019).

\section{SUMMARY OF THE PROPOSED CURRICULUM}

Highlights of Huygens' NIW-driven curriculum for seniors using the author's reference book "Causal Physics: Photon by Non-Interaction of Waves”, Taylor \& Francis, 2014 [1].

Chapter-1: Brief history:- We will present repeated discovery and neglect of NIW along the line of evolving physics [2]. Chapter-2: Establishing the value of the book:- Examples of prevailing contradictions and paradoxes that we currently accept in our fields of classical and quantum optical phenomena.

Chapter-3: Superposition Basics: This is the key transformational chapter. Use mathematics and experiments to underscore that the Superposition Principle (SP), the linear sum of wave amplitudes, $\sum_{n} E_{n}(t)$ ), is not an observable phenomenon in optics, unlike in radio wave domain. In the optical domain of high frequency, all the superposed EM waves simultaneously stimulate a quantum dipole (detecting molecule) to $\psi=\sum_{n} \chi(v) E_{n}(t), \chi(v)$ where $\chi(v)$ is the frequency-sensitive linear quantum dipolar polarizability of the detecting molecule. The observable signal is the 
nonlinear square modulus $\psi^{*} \psi$. All interpretations of optical physical phenomena must remain focused on observable $\psi^{*} \psi$ initiated by stimulation $\psi$.

Chapter-4: Diffraction:- "Borrow" "Hybrid Photon" model from Ch.9 and build diffraction integral emulating the Rayleigh-Somerfield diffraction integral. However, underscore that since all light consists of pulses, spontaneous, or stimulated, diffraction integral must accommodate this reality. What appears as CW light, in reality consist of quasiexponential pulses, which in-phase or out-of-phase.

Chapter-5: Spectrometry: We derive responses of Grating and Fabry-Perot spectrometers by propagating a time-finite causal pulse with its unique carrier frequency, instead of a non-causal and infinite Fourier frequency. We need to be very careful in tying the physical interpretations of measured line width with the internal atomic and molecular properties, unless the incident pulse width is at least $\sim R \lambda / c$. The center of the spectral fringe correctly determines "absolute" frequency of the incident light. The width of the fringe, for CW or pulsed light, represent instrumental fringe broadening. It does not represent physical presence of new frequencies that did not emanate from the original source.

Chapter-6: Coherence will be discussed as correlation of a pair of replicated pulses; the way we measure visibility of fringes. In light of NIW, it is meaningless to use the phrase "coherence of light". We should call it "correlation factor", faithfully representing the math and the experiments. Further, we will underscore that it is the detector's measuring time interval (or, time constant), which determine the fringe visibility. If we had Femto second streak camera, all pico second pulses will show fringes of decent visibility, depending upon the relative path delay.

Chapter-7: Mode lock lasers:- It is the mode locker with its time-finite quadratic response to become transparent gate, which determine the mode locked pulse shape. In the early stage of laser "turn on", the same process enforces phase locking between the cavity-allowed longitudinal modes. Real mode locked pulse shapes cannot be derived by using the square modulus of the linear sum of the "in-phase" longitudinal modes. Because, the physical process behind the evolution of pulses and phase locking are guided by the mode-locker. It is an involved evolutionary process.

Chapter-8: Dispersion:- Because of NIW, the traditional derivation of Group Velocity by summing two different phasesteady amplitude-waves, does not represent any natural physical process. Material dispersion, the refractive index dependent velocity of light, is a causal phenomenon. Examples will be analyzed in view of this understanding.

Chapter 9: Polarization:- We recognize that orthogonal polarizations "do not interfere". Our point is generalized NIW. This filed of optics has only minor confusions because Jones' Matrix method of analyzing polarized light correctly underscores that the polarization vectors vectors Ex's never "interfere” with Ey's.

Chapter-10: Model of light:- "Hybrid Photons" will be defined and justified as quantized energy packet (hv) at birth, which evolves into a classical quasi-exponential wave packet and keeps propagating as modeled by the HF diffraction integral. We will validate the model using spectrometry of various emissions and other justifications.

Chapter-11: Advanced Physics: Michelson-Morley experiment validated absence of "ether drag", not absence of etherlike medium. We define Complex Tension Field (CTF) as the "ether-like" medium and postulate particles as localized self-looped oscillations to accommodate absence CTF-drag. We will show the model's congruence with Quantum Mechanics. We will also briefly summarize the consequences of NIW in Relativity and Astrophysics (Doppler Effect, Cosmological Redshift, CMBR, etc.).

Chapter-12: Interaction Process Mapping Epistemology (IPM-E):- We will argue that one thousand years' of neglect of NIW has been able to continue because Physics did not pay close attention to discovering and visualizing the interaction processes that nature executes in maintaining the dynamic state of the evolving universe. Current evidence based science is the best approach, which can be characterized as - Measurable Data Modeling Epistemology (MDM-E). We will underscore the limitations of the prevailing MDM-E approach and show how to overcome those limitations by iteratively applying IPM-E.

\section{REFERENCES}

[1] C. Roychoudhuri, [Causal Physics: Photon by Non-Interaction of Waves], Taylor and Francis, (2014).

[2] C. Roychoudhuri, "Consequences of repeated discovery and benign neglect of Non-Interaction of Waves (NIW)", Proc. of SPIE Vol. 10452 1045215-1 (2017). doi: 10.1117/12.2266216. (2017).

[3] C. Huygens, [Treatise on Light], 1690. See http://www.gutenberg.org/ebooks/14725 for free download from Project Gutenberg edition (2005). 